\title{
Codificadores convolucionais generalizados com pacotes de informação de comprimento primo
}

\author{
Jorge Pedraza Arpasi
}

\begin{abstract}
Resumo-Códigos convolucionais generalizados sobre grupos arbitrários são necessários a partir do trabalho de Ungerboeck, o mesmo que trata sobre casamento entre bits codificados e constelações de sinais. $O$ conjunto das palavras de um código convolucional formam um sistema dinâmico que precisa ser bem comportado, isto é, ser controlável e observável. Um código que não seja controlável não pode ser um bom código. Neste trabalho mostramos que códigos convolucionais gerados pela extensão nãoabeliana $\mathbb{Z}_{p} \otimes S$, onde $\mathbb{Z}_{p}$ é o grupo cíclico $\{0,1,2, \ldots, p-1\}$, $p$ primo, não são controláveis ou tem distancia livre limitada por transições paralelas.
\end{abstract}

Palavras-Chave.- Códigos convolucionais generalizados, códigos de treliça, controle, $p$-grupos

\section{INTRODUÇÃO}

Forney e Trott, em [1], perceberam que o codificador convolucional com taxa de transmissão $\frac{1}{3}$ e memória 2 da Figura 1 pode ser descrito como uma máquina de estados $M=$ $\left(\mathbb{Z}_{2}, \mathbb{Z}_{2}^{2}, \mathbb{Z}_{2}^{3}, \nu, \omega\right)$. Este codificador, que em [2] é denotado por $(3,1,2)$, tem como alfabeto de entradas o conjunto $\mathbb{Z}_{2}=$ $\{0,1\}$, como saídas $\mathbb{Z}_{2}^{3}=\{000,100, \ldots, 111\}$, e como o conjunto dos estados $\mathbb{Z}_{2}^{2}=\{00,10,01,11\}$. Cada um dos con-

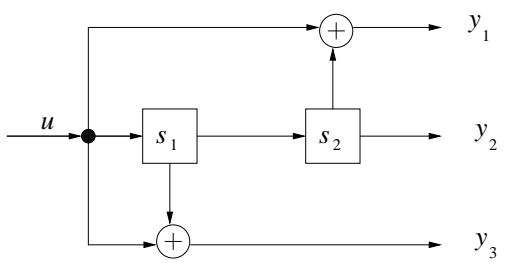

Fig. 1. O codificador de um código binário $(3,1,2)$

juntos $\mathbb{Z}_{2}, \mathbb{Z}_{2}^{2}, \mathbb{Z}_{2}^{3}$, com a operação soma módulo 2 , executada componente a componente, é um grupo. Por exemplo, para o caso $\mathbb{Z}_{2}^{3}$ temos $100+111=011,000+101=101,101+101=000$, etc. Isto mostra informalmente que $\mathbb{Z}_{2}^{3}$ com a soma módulo 2 possui as propriedades de clausura, associatividade, elemento neutro, e elemento inverso necessárias para ter estrutura de grupo. Mais ainda, estes grupos binários são grupos abelianos, pois a operação soma módulo 2 é comutativa. $\mathrm{O}$ conjuntos $\mathbb{Z}_{2}^{2}=\mathbb{Z}_{2} \times \mathbb{Z}_{2}$, e $\mathbb{Z}_{2}^{3}=\mathbb{Z}_{2} \times \mathbb{Z}_{2} \times \mathbb{Z}_{2}=\mathbb{Z}_{2} \times \mathbb{Z}_{2}^{2}$ são produtos cartesianos chamados de grupos produto direto. A dinâmica do codificador da Figura 1 pode ser descrita pelo mapeamento do próximo estado $\nu: \mathbb{Z}_{2} \times \mathbb{Z}_{2}^{2} \rightarrow \mathbb{Z}_{2}^{2}$ e pelo mapeamento codificador $\omega: \mathbb{Z}_{2} \times \mathbb{Z}_{2}^{2} \rightarrow \mathbb{Z}_{2}^{3}$ sendo que ambos são

Departamento de Ciências Exatas e Engenharias, Universidade Regional Integrada - URI, Frederico Westphalen, RS. Email: arpasi@fw.uri.br homomorfismos de grupos definidos por $\nu\left(u, s_{1} s_{2}\right)=\left(u, s_{1}\right)$ e $\omega\left(u, s_{1} s_{2}\right)=\left(u+s_{2}, s_{2}, u+s_{1}\right)$. Em geral, para qualquer taxa de transmissão $\frac{k}{n}$ e qualquer memória $m$, um codificador convolucional binário pode ser definido como a máquina de estados $M=\left(\mathbb{Z}_{2}^{k}, \mathbb{Z}_{2}^{m}, \mathbb{Z}_{2}^{n}, \nu, \omega\right)$ onde $\nu: \mathbb{Z}_{2}^{k} \times \mathbb{Z}_{2}^{m} \rightarrow \mathbb{Z}_{2}^{m}$ é o homomorfismo do próximo estado e $\omega: \mathbb{Z}_{2}^{k} \times \mathbb{Z}_{2}^{m} \rightarrow \mathbb{Z}_{2}^{n}$ é o homomorfismo codificador. Como $k+m \geq m$, temos que $\nu$ é homomorfismo sobrejetor, enquanto que, para evitar códigos catastróficos o homomorfismo codificador $\omega$ deve ser injetor, isto é, $n \geq k+m$ necessariamente.

Desde o trabalho de Ungerboeck [3], onde o problema central era o casamento entre os pacotes de bits codificados que formam o grupo binário $\mathbb{Z}_{2}^{n}$, e uma constelação de sinais $S$, houve a necessidade de ampliar o conceito de códigos convolucionais. Então foram introduzidos conceitos de códigos convolucionais generalizados sobre grupos (group codes) [1], e casamento entre grupos e pontos discretos de um espaço Euclidiano, [4]. Os grupos referidos nesta generalização podem ser até grupos não-abelianos. Em [4], Loeliger mostra teoricamente que para um canal AWGN dado, usando códigos convolucionais abelianos sua capacidade de transmissão é limitada superiormente pela capacidade de um canal AWGN com modulação PSK (Phase Shift Keying). Assim, canais usando códigos convolucionais sobre grupos não-abelianos poderiam superar esta limitação.

Um conceito fundamental da álgebra que é necessário para definir codificadores convolucionais generalizados, e portanto códigos convolucionais generalizados, é a definição de extensão de grupos que introduziremos na Seção II, onde será mostrado que produto direto de grupos é um caso particular de extensão de grupos. Na seção III definiremos codificadores convolucionais generalizados e a abordagem dos códigos convolucionais como sequências bi-infinitas que para serem bons códigos precisam ser bem comportados quando identificados como sistemas dinâmicos [5], [4], [1], [6] Um sistema dinâmico bem comportado precisa ser observável e controlável [7]. Na Seção IV enfocaremos os codificadores convolucionais sobre a extensão não-abeliana $\mathbb{Z}_{p} \otimes S$, onde $p$ é primo e $\mathbb{Z}_{p}$ é o grupo cíclico $\mathbb{Z}_{p}=\{0,1,2, \ldots, p-1\}$ e mostraremos que um código convolucional, definido sobre esta extensão, não é controlável ou tem distância livre limitada por transições paralelas.

\section{EXTENSÃO DE GRUPOS}

Definições padrão de extensão de grupos são dados em [8], [9], entre outros. Para a definição que daremos a seguir, ao elemento neutral(identidade) de um grupo genérico $G$ 
denotaremos por $e$, a notação $N \triangleleft G$ significa $N$ é um subgrupo normal de $G$, enquanto que $H \cong K$ será a notação para um isomorfismo entre $H$ e $K$.

Definição 1: Uma extensão de um grupo $U$ por outro $S$ é um grupo $G$ que possui um subgrupo normal $N \triangleleft G$, tal que $N \cong U$ e $\frac{G}{N} \cong S$. 。

Dado que cada grupo $G$, pelo menos, possui seus subgrupos normais triviais, então cada grupo $G$ sempre é a extensão de algum grupo $U$ por grupo $S$, à qual denotaremos por $U \otimes S$. Isto significa que cada elemento $g \in G$ pode ser "factorado"ou decomposto como um único par ordenado $(u, s), u \in U \mathrm{e}$ $s \in S$. A construção desta fatoração é baseada na escolha dos isomorfismos $\psi: S \rightarrow \frac{G}{N}$ e $v: N \rightarrow U$ referidos na Definição 1 , e de um levantamento $l: \frac{G}{N} \rightarrow G$, chamado também escolha de representante de classe, tal que $l(N)=e$. Com estas escolhas são definidos os mapeamentos $\varsigma: S \times S \rightarrow$ $U$ e $\phi: S \rightarrow A u t(U)$

$$
\varsigma(s, t)=v\left[l(\psi(s)) \cdot l(\psi(t)) \cdot(l(\psi(s t)))^{-1}\right],
$$

e

$$
\phi(s)(u)=v\left[l(\psi(s)) \cdot v^{-1}(u) \cdot(l(\psi(s)))^{-1}\right] .
$$

Então $U \otimes S$ com a operação

$$
\left(u_{1}, s_{1}\right) *\left(u_{2}, s_{2}\right)=\left(u_{1} \cdot \phi\left(s_{1}\right)\left(u_{2}\right) \cdot \varsigma\left(s_{1}, s_{2}\right), s_{1} s_{2}\right)
$$

é um grupo isomorfo com $G$.

O produto semidireto $U \rtimes S$ é um caso particular de extensão, onde $\varsigma(s, t)=e \in U$ para quaisquer $s, t \in S$. Por outro lado, o produto direto é um caso particular de extensão, onde $\varsigma(s, t)=$ $e \in U$ para quaisquer $s, t \in S$, e $\phi(s)$ é o automorfismo identidade para qualquer $s \in S$. Portanto a extensão $U \otimes$ $S$ é uma generalização do produto direto $U \times S$, conforme dizeramos linhas acima.

Exemplo 1: Considere o grupo $\mathbb{Z}_{2}^{3}=\left\{\left(x_{1}, x_{2}, x_{3}\right) ; x_{i} \in\right.$ $\left.\mathbb{Z}_{2}\right\}$. Este grupo abeliano pode ser decomposto como o produto direto $\mathbb{Z}_{2} \times \mathbb{Z}_{2}^{2}$ e portanto uma extensão de $\mathbb{Z}_{2}$ por $\mathbb{Z}_{2}^{2}$. 。

Exemplo 2: Considere o grupo das simetrias do quadrado, $D_{8}=\left\{R_{0}, R_{\frac{\pi}{2}}, R_{\pi}, R_{\frac{3 \pi}{2}}, d_{1}, d_{2}, H, V\right\}$, onde $R_{i \frac{\pi}{2}}$ é uma rotação do quadrado, no sentido anti-horário, de $i \frac{\pi}{2}$ radianos, $d_{1}$, e $d_{2}$ são reflexões referidas às diagonais, e $H, V$ são as reflexões horizontal e vertical respectivamente.

Um subgrupo normal é $N=\left\{R_{0}, R_{\pi}\right\} \cong \mathbb{Z}_{2}$, e para o grupo dos cosets $\frac{D_{8}}{N}$ temos $\mathbb{Z}_{2}^{2} \cong \frac{D_{8}}{N}$. Na Tabela I temos as escolhas de $\psi, v$, e $l$. Por exemplo, $\psi(10)=$ $\left\{R_{\pi / 2}, R_{3 \pi / 2}\right\}, v\left(R_{0}\right)=0$, e $l\left(\left\{R_{\pi / 2}, R_{3 \pi / 2}\right\}\right)=R_{\pi / 2}$. Com isto, a operação de grupo para $D_{8}=\mathbb{Z}_{2} \otimes \mathbb{Z}_{2}^{2}$ é dada por $\left(i_{1}, i_{2} i_{3}\right)\left(j_{1}, j_{2} j_{3}\right)=\left(i_{1}+j_{1}+\varsigma\left(i_{2} i_{3}, j_{2} j_{3}\right), i_{2} i_{3}+j_{2} j_{3}\right)$. Por exemplo; $(0,10)(1,10)=(0+1+\varsigma(10,10), 10+10)=(1+$ $\left.v\left(l(\psi(10)) l(\psi(10))(l(\psi(00)))^{-1}\right), 00\right)=\left(1+v\left(R_{\frac{\pi}{2}} R_{\frac{\pi}{2}}\right), 00\right)$ $=\left(R_{0}, 00\right)$. 。

Notemos que o resultado de $\left(u_{1}, s_{1}\right) \cdot\left(u_{2}, s_{2}\right)$, da operação acima (3), é $\left(u^{\prime}, s_{1} s_{2}\right)$ para algum $u^{\prime}$ e onde $s_{1} s_{2}$ é a operação do grupo $S$. Esta propriedade nos deixa livres da preocupação do cálculo exato do produto de múltiplos pares. Por exemplo na prova de alguns Lemas será suficiente saber que $\left(u^{\prime}, s_{1} s_{2} \ldots s_{n}\right)$, é o par resultante do produto múltiplo $\left(u_{1}, s_{1}\right) \cdot\left(u_{2}, s_{2}\right) \cdot\left(u_{3}, s_{3}\right) \ldots\left(u_{n}, s_{n}\right)$, onde $u^{\prime}$ é algum elemento de $U$. Analogamente, $(u, s)^{n}=\left(u^{\prime}, s^{n}\right)$ para algum $u^{\prime} \in U$.

\begin{tabular}{ccccc}
\hline $\mathbb{Z}_{2}^{2}$ & $\psi$ & $\frac{D_{8}}{N}$ & $l$ & $D_{8}$ \\
\hline 00 & $\mapsto$ & $\left\{R_{0}, R_{\pi}\right\}$ & $\mapsto$ & $R_{0}$ \\
10 & $\mapsto$ & $\left\{R_{\pi / 2}, R_{3 \pi / 2}\right\}$ & $\mapsto$ & $R_{\pi / 2}$ \\
01 & $\mapsto$ & $\left\{d_{1}, d_{2}\right\}$ & $\mapsto$ & $d_{1}$ \\
11 & $\mapsto$ & $\{H, V\}$ & $\mapsto$ & $H$ \\
\hline & & $\downarrow v$ & & \\
& \multicolumn{5}{c}{0,1} & & \\
\hline \multicolumn{5}{c}{$\mathbb{Z}_{2}$} \\
\end{tabular}

\section{TABELA I}

ESCOLHAS DOS ISOMORFISMOS $\psi$ E $v$, E O LEVANTAMENTO $l$ PARA O EXEMPLO 2

\section{CODIFICADORES E CÓDIGOS CONVOLUCIONAIS GENERALIZADOS}

Definição 2: Um codificador homomorfo generalizado é uma máquina $M=(U, Y, S, \omega, \nu)$, onde o alfabeto de entrada $U$, o alfabeto de saída $Y$, e o conjunto dos estados da máquina $S$ são grupos tais que o mapeamento do próximo estado $\nu$ é um homomorfismo sobrejetor e o mapeamento codificador $\omega$ é um homomorfismos injetor, ambos definidos assim;

$$
\left\{\begin{array}{l}
\nu: U \otimes S \rightarrow S \\
\omega: U \otimes S \rightarrow G
\end{array}\right.
$$
○

Exemplo 3: Considere o grupo produto direto $\mathbb{Z}_{2}^{3}=$ $\left\{\left(x_{1}, x_{2}, x_{3}\right) ; x_{i} \in \mathbb{Z}_{2}\right\}$ (Exemplo 1). Definindo $\nu$ : $\mathbb{Z}_{2} \times \mathbb{Z}_{2}^{2} \rightarrow \mathbb{Z}_{2}^{2}$ como sendo $\nu\left(u, s_{1}, s_{2}\right)=\left(u, s_{1}\right)$ e $\omega:$ $\mathbb{Z}_{2} \times \mathbb{Z}_{2}^{2} \rightarrow \mathbb{Z}_{2}^{3}$ por $\omega\left(u, s_{1}, s_{2}\right)=\left(u+s_{2}, s_{2}, u+s_{1}\right) ;$ temos um codificador $M=\left(\mathbb{Z}_{2}, \mathbb{Z}_{2}^{2}, \mathbb{Z}_{2}^{3}, \nu, \omega\right)$ que gera o codificador binário da Figura 1.
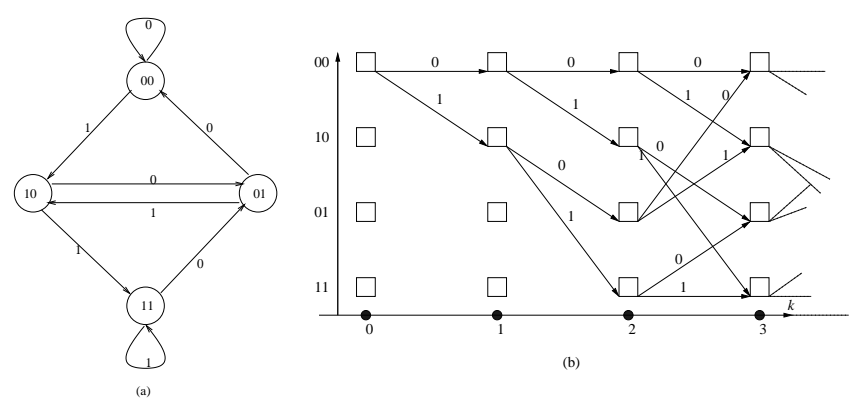

Fig. 2. Representação gráfica do código convolucional $(3,1,2)$ : (a) Diagrama de estados estático conforme Teoria dos grafos, (b) Dinâmica da Treliça conforme Teoria de códigos corretores de erros.

Supondo que o estado inicial do codificador $M$ seja 00 , temos que a sequência de bits de entrada $1,1,0,1,0,0,1,1,1,0, \ldots$ gera de maneira única a sequência de estados $10,11,01,10,01,00,10,11,11,01, \ldots$ e a sequência de bits codificados $101,100,111,011,001,110,101,100,010,111, \ldots$ 
seguinte forma;

$$
\begin{array}{ll|l}
\nu(0,00)=10 & \omega(0,00)=101 \\
\nu(1,10)=11 & \omega(1,10)=100 \\
\nu(0,11)=01 & \omega(0,11)=111 \\
\nu(1,01)=10 & \omega(1,01)=011 \\
\nu(0,10)=01 & \omega(0,10)=001 \\
\nu(0,01)=00 & \omega(0,01)=110 \\
\nu(1,00)=10 & \omega(1,00)=101 \\
\nu(1,10)=11 & \omega(1,10)=100 \\
\nu(1,11)=11 & \omega(1,11)=010 \\
\nu(0,11)=01 & \omega(0,11)=111
\end{array}
$$

$\circ$

Exemplo 4: Considere o grupo das simetrias do quadrado, $D_{8}$ (Exemplo 2). Considere o codificador dado por $\omega(a, b c)=$ $(a, b c)$ e $\nu(a, b c)=b c$ temos um codificador $M=$ $\left(\mathbb{Z}_{2}, \mathbb{Z}_{2}^{2}, D_{8}, \nu, \omega\right)$.

O codificador para esta extensão não-abeliana, não é implementável como um circuito registrador de deslocamentos. No entanto ele possui um diagrama de estados e um diagrama de treliça mostrados na Figura 3. o
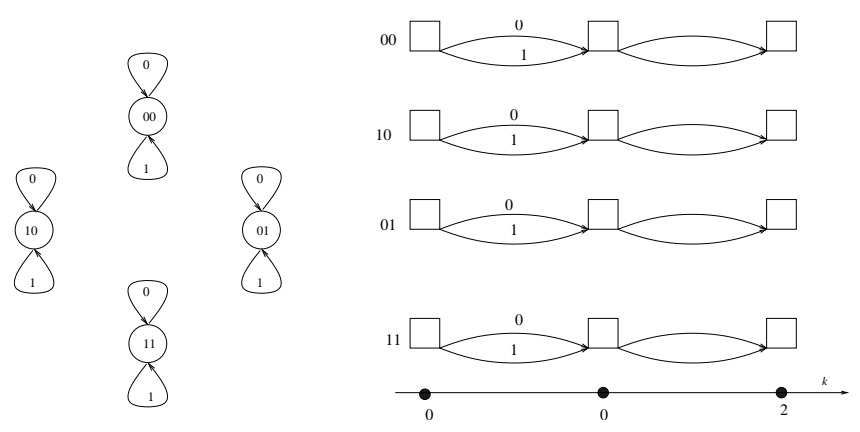

Fig. 3. (a) Grafo disconexo do codificador 4 (b)Treliça

Seja $\mathcal{C}$ o código binário produzido pelo codificador da Figura 1 e Exemplo 1, temos que $\mathcal{C}$ é um conjunto de sequências de pacotes de três bits, por exemplo $\{101,100,111,011,001,110,101,100,010,111, \ldots\} \in$ $\mathcal{C}$. Então, considerando o produto direto infinito $\left(\mathbb{Z}_{2}^{3}\right)^{\mathbb{N}}=$ $\mathbb{Z}_{2}^{3} \times \mathbb{Z}_{2}^{3} \times \ldots$ temos $\mathcal{C}$ é um subgrupo de $\left(\mathbb{Z}_{2}^{3}\right)^{\mathbb{N}}$. Sob o ponto de vista dos sistemas dinâmicos, $\mathcal{C}$ pode ser descrito como um sistema invariante no tempo, pois para cada índice em $\mathbb{N}$, o grupo $\mathbb{Z}_{2}^{3}$ é repetido. Por isso, também, estes códigos são chamados códigos convolucionais invariantes no tempo.

Um código convolucional generalizado é indexado sobre os inteiros $\mathbb{Z}$.

Definição 3: Considere uma família de grupos $\left\{G_{k}\right\}_{k \in \mathbb{Z}}$ e o produto direto, indexado em $\mathbb{Z}, \mathcal{G}=\cdots \times G_{k-1} \times$ $G_{k} \times G_{k+1} \times \ldots$ Temos que cada elemento deste produto é um sequência $\left\{g_{k}\right\}_{k \in \mathbb{Z}}, g_{k} \in G_{k}$, e com as operações de grupo induzidas componente a componente sobre cada $G_{k}, \mathcal{G}$ também é grupo. Então, um código convolucional generalizado $\mathcal{C}$, é um subgrupo de $\mathcal{G}$.

Se para cada $G_{k}$ temos que $G_{k}=G$, então temos $\mathcal{G}=G^{\mathbb{Z}}=$ $\ldots \times G \times G \times G \times \ldots$ E neste caso temos que um subgrupo
$\mathcal{C}$ de $\mathcal{G}$ chamado de código convolucional invariante no tempo [5], [4], [1], [6].

Uma sequência $\left\{\mathbf{c}_{k}\right\}_{k \in \mathbb{Z}} \in \mathcal{C}$ é chamada de palavra-código. Dados dois inteiros $i, j$, com $i \leq j$, usaremos as notações $[i, j],[i, j),(i, j]$, e $(i, j)$ para intervalos inteiros. Por exemplo, $[i, j]=\{i, i+1, \ldots, j-1, j\},[i, j)=\{i, i+1, \ldots, j-1\}$, e assim por diante. Esta notação também funciona em em conjuntos discretos infinitos tal como $\{k \in \mathbb{Z} ; k \leq j\}=$ $(-\infty, j]$. Com isto a projeção de uma palavra-código $\left\{\mathbf{c}_{k}\right\}_{k \in \mathbb{Z}}$ sobre o conjunto de índices $[i, j]$ é denotado por $\left.\{\mathbf{c}\}\right|_{[i, j]}=$ $\left\{\mathbf{c}_{i}, \mathbf{c}_{i+1}, \ldots, \mathbf{c}_{j}\right\}$.

Dadas duas palavras-código $\left\{\mathbf{c}_{1 k}\right\}_{k \in \mathbb{Z}},\left\{\mathbf{c}_{\boldsymbol{2}}\right\}_{k \in \mathbb{Z}} \in \mathcal{C}$, uma concatenação de $\left\{\mathbf{c}_{\mathbf{1}_{k}}\right\}_{k \in \mathbb{Z}}$ e $\left\{\mathbf{c}_{\mathbf{2}}\right\}_{k \in \mathbb{Z}}$ no instante $j$ é uma palavra código $\left\{\left(\mathbf{c}_{\mathbf{1}} \wedge_{j} \mathbf{c}_{\mathbf{2}}\right)_{k}\right\}_{k \in Z}$ definida como $\left(\mathbf{c}_{\mathbf{1}} \wedge_{j} \mathbf{c}_{\mathbf{2}}\right)_{k}=$ $\left\{\begin{array}{l}\left.\mathbf{c}_{\boldsymbol{1}_{k}}\right|_{(-\infty, j)} ; k<j \\ \left.\mathbf{c}_{\mathbf{2} k}\right|_{[j,+\infty)} ; k \geq j .\end{array}\right.$

Se $L$ é um inteiro maior do que um, então o código de grupo $\mathcal{C}$ é dito $L$-controlável quando para dadas duas palavras $\mathbf{c}_{1}$ e $\mathbf{c}_{2}$, existir uma terceira palavra $\mathbf{c}_{3}$ e um inteiro $k$ tal que a concatenação $\mathbf{c}_{\boldsymbol{1}} \wedge_{k} \mathbf{c}_{\boldsymbol{3}} \wedge_{k+L} \mathbf{c}_{\mathbf{2}}$ é uma palavra do código de grupo $\mathcal{C}$. [6], [5]. É dito que um número natural $l>1$ é o índice de controlabilidade do código $\mathcal{C}$ quando $l=$ $\min \{L ; \mathcal{C}$ é $L$-controlável $\}$. Qualquer código de grupo que tenha uma aplicação prática em transmissão o armazenamento de dados precisa ter um índice de controlabilidade.

Definição 4: Um código de grupo $\mathcal{C}$ é dito controlável quando existir um inteiro $l>1$ tal que $l$ é o índice de controle de $\mathcal{C}$. o

Em [1] tem sido provado que códigos invariantes no tempo podem ser gerados por um codificador convolucional generalizado. Conforme foi notado em [5], [10], considerando códigos convolucionais generalizados como sistemas dinâmicos os codificadores convolucionais generalizados são a realização destes códigos.

A seção de treliça é o conjunto de arestas $(s, \omega(u, s), \nu(u, s)) \in S \times Y \times S, \quad$ e $\quad$ se pode provar que o conjunto de todas as arestas $B=$ $\{(s, \omega(u, s), \nu(u, s)) ;(u, s) \in U \otimes S\}$ é um grupo que é isomorfo a $U \otimes S$

O código de grupo $\mathcal{C}$ gerado pelo codificador da Definição 2 não será controlável se existirem dois estados $s$ e $s^{\prime}$ tais que $s \neq \nu\left(u_{n}, \nu\left(u_{n-1}, \nu\left(u_{n-2}, \ldots, \nu\left(u_{2}, \nu\left(u_{1}, s^{\prime}\right)\right) \ldots\right)\right)\right)$, para quaisquer sequência de $\left\{u_{i}\right\}_{i=1}^{n}$ entradas.

Exemplo 5: Para o caso do codificador binário do Exemplo 3 , Figura 1, temos que o código resultante é controlável conforme pode ser visualizado na Figura 2. Para o caso do codificador do Exemplo 4. Por uma simples inspeção visual da Figura 3, podemos concluir que o código não é controlável. Mais ainda, podemos mostrar que para todas as outras extensões dos subgrupos normais $\left\{R_{0}, R_{\pi / 2}, R_{\pi}, R_{3 \pi / 2}\right\}$, $\left\{R_{0}, R_{\pi}, H, V\right\}$, e $\left\{R_{0}, R_{\pi}, d_{1}, d_{2}\right\}$ não é possível construir grafos conexos, o que significa que não existe homomorfismos sobrejetores $\nu: D_{8}=U \otimes S \rightarrow S$ tais que produzam codificadores controláveis. $\circ$

Dado um codificador da Definição 2, considere a família de subconjuntos $\left\{S_{i}\right\}$, do grupo dos estados $S$ definidos recursivamente por; 


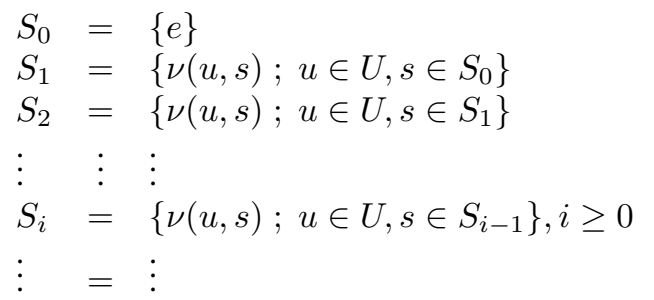

Teorema 1: Algumas propriedades de $\left\{S_{i}\right\}$;

1) Cada $S_{i}$ é um subgrupo de $S$

2) $S_{i-1}$ é normal em $S_{i}$, para cada $i=1,2, \ldots$

3) Se $S_{i-1}=S_{i}$ então $S_{i}=S_{i+1}$.

4) Se o código é controlável então $S=S_{k}$ para algum $k \in \mathbb{N}$.

\section{Prova.-}

1) Considere $r, s \in S_{i}$, como $\nu$ é sobrejetor, existem $\left(u_{1}, s_{1}\right)$ e $\left(u_{2}, s_{2}\right)$ com $s_{1}, s_{2} \in S_{i-1}$ e $u_{1}, u_{2} \in U$ tal que $r=\nu\left(u_{1}, s_{1}\right)$ e $s=\nu\left(u_{2}, s_{2}\right)$. Daí, $s r=$ $\nu\left(u_{3}, s_{1} s_{2}\right), u_{3} \in U$ e assim $s r \in S_{i}$.

2) Claramente $S_{0} \triangleleft S_{1}$. Para $i>1$, suponha $S_{j-1} \triangleleft S_{j}$, para cada $j \leq i$. Dados $s \in S_{i+1}$ e $r \in S_{i}$, considere s.r.s $s^{-1}$ $=\nu\left(u, s_{1}\right) \cdot \nu\left(v, r_{1}\right) \cdot \nu\left(u, s_{1}\right)^{-1}$, onde $s_{1} \in S_{i}, r_{1} \in S_{i-1}$, $u, v \in U$. Daí, s.r.s $s^{-1}=\nu\left(u_{1}, r_{1} . s_{1} \cdot r_{1}^{-1}\right) \in S_{i}$, pois $r_{1} \cdot s_{1} \cdot r_{1}^{-1} \in S_{i-1}$.

3) Dado $s \in S_{i+1}$ existem $r \in S_{i}$ e $u \in U$ tais que $\nu(u, r)=s$. Como $S_{i}=S_{i-1}, r \in S_{i-1}$. Portanto $\nu(u, r)=s \in S_{i}$.

4) Em caso contrário, existem $s \in S_{k}$ e $s^{\prime} \in S$ tais que $s^{\prime} \neq \nu\left(u_{n}, \nu\left(u_{n-1}, \nu\left(u_{n-2}, \ldots, \nu\left(u_{2}, \nu\left(u_{1}, s\right)\right) \ldots\right)\right)\right)$, para qualquer sequência $\left\{u_{i}\right\}_{i=1}^{n}$ de entradas.

O conjunto de transições $(s, \omega(u, s), \nu(u, s)) \in(S \times$ $Y \times S)$ de um código de grupo invariante no tempo é um grafo orientado cujos conjunto de vértices é o grupo de estados $S$ e as arestas é o conjunto das triplas $(s, \omega(u, s), \nu(u, s))$. Em cada tripla, o estado $s$ é o ponto de partida da aresta, enquanto que $\nu(u, s)$ é o ponto final da aresta. Com a operação componente a componente $\left(s_{1}, \omega\left(u_{1}, s_{1}\right), \nu\left(u_{1}, s_{1}\right)\right) *\left(s_{2}, \omega\left(u_{2}, s_{2}\right), \nu\left(u_{2}, s_{2}\right)\right)=$ $\left(s_{1} s_{2}, \omega\left(\left(u_{1}, s_{1}\right)\left(u_{2}, s_{2}\right)\right), \nu\left(\left(u_{1}, s_{1}\right)\left(u_{2}, s_{2}\right)\right)\right)$ este conjunto de arestas orientadas, será denotada por $E$. Dentro da área da Teoria dos Códigos Corretores de Erros, as arestas são melhor conhecidas como transições e um exemplo de estas duas representações gráficas é mostrada nas Figuras 2 e 3

Desde que $\omega$ é injetora, o mapeamento $\Psi: U \otimes S \rightarrow E$ definido por,

$$
\Psi(u, s)=(s, \omega(u, s), \nu(u, s))
$$

é um isomorfismo de grupos.

Lema 1: Considere o codificador $\omega, \nu$, e $U \otimes S$ da Definição 2. Suponha $U \otimes S$ não-abeliano. Sejam $E^{+}$e $E^{-}$subconjuntos do grupo seção de treliça $E$ tal que $E^{+}=$ $\{(e, \omega(u, e), \nu(u, e) ; u \in U\}$, as arestas saindo do estado neutro $\{e\}$, e $E^{-}=\{(s, \omega(u, s), \nu(u, s) ; \nu(u, s)=e\}$, as arestas chegando no estado neutro $\{e\}$ então;
1) Ambos $E^{+}$e $E^{-}$são subgrupos normais de $E$, com $|U|=\left|E^{+}\right|=\left|E^{-}\right|$

2) Os grupos quocientes $\frac{E}{E^{+}}$e $\frac{E}{E^{-}}$são isomorfos e $\frac{E}{E^{+}} \cong$ $\frac{E}{E^{-}} \cong S$.

3) O número de arestas saindo/chegando de/em qualquer estado $s$ é $|U|=\left|E^{+}\right|=\left|E^{-}\right|$

\section{Prova.-}

1) Imediato

2) O mapeamento de $S$ a $\frac{E}{E^{+}}$dado por $s \mapsto$ $(s, \omega(u, s), \nu(u, s)) E^{+}$, é um homomorfismo bijetor, daí $\frac{E}{E^{+}} \cong S$. Por outro lado, a projeção $(s, \omega(u, s), \nu(u, s)) \mapsto \nu(u, s)$, de $E$ a $S$, é um homomorfismo sobrejetor com kernel $E^{-}$, pelo Teorema fundamental dos homomorfismos, $\frac{E}{E^{-}} \cong S$

3) Considere a transição $t_{0}=(s, \omega(e, s), \nu(e, s))$ saindo de um estado qualquer $s$, e o coset $t_{0} E^{+}=t_{0}\{\Psi(u, e) ; u \in$ $U\}=\{\Psi(u, s) ; u \in U\}$ que é o conjunto das transições saindo de $s$ e que possui $\left|E^{+}\right|$arestas. Analogamente considerando $s E^{-}$podemos mostrar que as transições chegando no estado $s$ tem cardinalidade $\left|E^{-}\right|$.

Definição 5: Dado um grupo $G$, o subgrupo dos comutadores de $G$ é definido por $G^{\prime}=\left\{a b a^{-1} b^{-1} ; a, b \in G\right\}$

Definição 6: Duas arestas diferentes $\left(s_{1}, \omega\left(u_{1}, s_{1}\right), \nu\left(u_{1}, s_{1}\right)\right)$ e $\left(s_{2}, \omega\left(u_{2}, s_{2}\right), \nu\left(u_{2}, s_{2}\right)\right)$ são ditas paralelas se $s_{1}=s_{2}$ e $\nu\left(u_{1}, s_{1}\right)=\nu\left(u_{2}, s_{2}\right)$ e $\omega\left(u_{1}, s_{1}\right) \neq \omega\left(u_{2}, s_{2}\right)$

Quando o grupo seção de treliça $E$ não possui transições paralelas, qualquer arco pode ser representado, de maneira unívoca, por um par $(s, \nu(u, s))$, onde o estado $s$ é o vértice de saída, e $\nu(u, s)$ é o vértice de chegada. O seguinte Lema é uma versão para codificadores homomorfos do Teorema 4 de [11]

Lema 2: Considere o codificador $\omega, \nu$, e $U \otimes S$ da Definição 2. Sejam $H^{+}$e $H^{-}$subconjuntos de $U \otimes S$ tais que $H^{+}=$ $U \otimes\{e\}=\{(u, e) ; u \in U\}$ e $H^{-}=\operatorname{Ker}(\nu)=$ $\{(u, s) ; \nu(u, s)=e\}$, então;

1) $H^{+} \cong E^{+}$e $H^{-} \cong E^{-}$,

2) Ambos $H^{+}$e $H^{-}$são subgrupos normais de $U \otimes S$,

3) Se $H^{+} \cap H^{-} \neq\{(e, e)\}$ então a seção de treliça $E$ possui transições paralelas,

4) Se $U \otimes S$ é não-abeliano e o grupo de estados $S$ é abeliano então $E$ possui transições paralelas

\section{Prova.-}

1) Temos $E^{+}=\Psi\left(H^{+}\right)$e $E^{+}=\Psi\left(H^{+}\right)$, com $\Psi$ definido pela equação (5).

2) Imediato.

3) Existe $(u, e) \in H^{+} \cap H^{-}$, com $u \neq e$ tal que $\nu(u, e)=$ $e$, pois $\Psi$ de (5) é bijetor, $\omega(u, e) \neq e$. Portanto, as transições $(e, \omega(e, e), \nu(e, e))$ e $(e, \omega(u, e), \nu(u, e))$ são paralelas.

4) O fato do grupo dos estados $S$ ser abeliano implica que $\frac{G}{H^{+}} \cong \frac{G}{H^{-}}$são grupos quocientes abelianos. Então o subgrupo dos comutadores $(U \otimes S)^{\prime}$ é um subgrupo de $H^{+} \cap H^{-}$[8]. Mas $U \otimes S$ é não abeliano, então $(U \otimes S)^{\prime} \neq\{(e, e)\}$. Portanto do anterior item 2,B tem transições paralelas. 


\section{CODIFICADOR HOMOMORFO DEFINIDO EM $\mathbb{Z}_{p} \otimes S$ COM $p$ PRIMO}

A pesar da sua aparente simplicidade, ainda não existe uma classificação geral para $p$-grupos. Somente os $p$-grupos com ordem menor ou igual a $p^{6}$ tem sido completamente classificados, quando $p \geq 3$, [12]. E para o caso $p=2$, uma classificação completa tem sido feita para grupos com ordem $\leq 2^{8}$, [13], [14]. Esta classificação dos 2-grupos tem sido implementado em alguns softwares como o GAP, [14], que inclui em sua biblioteca todos os grupos de ordem 256. Os grupos cíclicos $\mathbb{Z}_{p}=\{0,1,2, \ldots, p-1\}$, onde a operação de grupo é dada por $i+j$ modulo $p$, são os exemplos mais simples de $p$-grupos. Os resultados acerca de codificadores homomorfos generalizados, envolvendo $\mathbb{Z}_{p}$ como grupo de informação, são válidos para qualquer $p$-grupo independentemente da existência de sua classificação.

Lema 3: Seja $\mathbb{Z}_{p} \otimes S$ uma extensão que é um $p$-grupo. Se $\mathbb{Z}_{p} \otimes S_{0} \subset\left(\mathbb{Z}_{p} \otimes S\right)^{\prime}$, então $\mathbb{Z}_{p} \otimes S_{i} \subset\left(\mathbb{Z}_{p} \otimes S\right)^{\prime}$, e $S_{i} \subset S^{\prime}$, para cada $i \geq 1$.

Prova.- Desde que $\nu$ é um homomorfismo de grupos, a imagem $\nu\left(\mathbb{Z}_{p} \otimes S_{0}\right)=S_{1}$ esta contida no subgrupo de comutadores $S^{\prime}$ de $S$. Se $S_{1}=S_{0}$ o Lema se cumpre trivialmente, (Figura 4 (a)).Se $S_{1} \neq S_{0}$, pelo teorema longo dos comutadores de [15], existem $s \in\left(S_{1}-S_{0}\right)$ e $a_{1}, a_{2}, \ldots, a_{t} \in S$ tais que $s=a_{1} a_{2} \ldots a_{t} a_{1}^{-1} a_{2}^{-1} \ldots a_{t}^{-1}$. Agora considere $u \in \mathbb{Z}_{p}$ e $\left\{u_{1}, u_{2}, \ldots, u_{t}\right\} \subset \mathbb{Z}_{p}$ tal que $(u, s)=$ $\left(u_{1}, a_{1}\right)\left(u_{2}, a_{2}\right) \ldots\left(u_{t}, a_{t}\right)\left(u_{1}, a_{1}\right)^{-1}\left(u_{2}, a_{2}\right)^{-1} \ldots\left(u_{t}, a_{t}\right)^{-1}$. temos $(u, s) \in\left(\mathbb{Z}_{p} \otimes S\right)^{\prime}$ e $(u, s) \notin \mathbb{Z}_{p} \otimes S_{0}$. Portanto $\mathbb{Z}_{p} \otimes S_{1} \subset\left(\mathbb{Z}_{p} \otimes S\right)^{\prime}$ (Figura 4 (b)).

De novo, e desde que $\nu$ é um homomorfismo de grupos, $\nu\left(\mathbb{Z}_{p} \otimes S_{1}\right)=S_{2}$ esta contido no subgrupo dos comutadores $S^{\prime}$ de $S$. Então com argumentos muito semelhantes, podemos provar que se $S_{2} \neq S_{1}$, então $\left(\mathbb{Z}_{p} \otimes S_{2}\right) \subset\left(\mathbb{Z}_{p} \otimes S\right)^{\prime}$ e $\nu\left(\mathbb{Z}_{p} \otimes S_{2}\right)=S_{3} \subset S^{\prime}$. Continuando da mesma maneira teremos $\left(\mathbb{Z}_{p} \otimes S\right)^{\prime}$ e $S_{i} \subset S^{\prime}$, para qualquer $i \geq 1$.

Lema 4: Seja $\mathbb{Z}_{p} \otimes S$ uma extensão que é um $p$-grupo. Considere os subgrupos $\left\{S_{i}\right\}$ definidos na equação (4). Então, $S_{i}$ é abeliano ou $S_{i} \subset S^{\prime}$, para cada $i$.

Prova.- Desde que $S_{1}$ é cíclico e $S_{2}$ tem ordem menor ou igual a $p^{2}$, temos que ambos $S_{1}$ e $S_{2}$ são abelianos. Então, seja $i \geq 2$ tal que $S_{1}, S_{2}, \ldots, S_{i}$ são todos abelianos com $S_{i+1}$ não abeliano. Então, existem $s_{1}, s_{2} \in S_{i+1}$ tais que $s_{1} s_{2} \neq s_{2} s_{1}$. Também deve existir $u_{1}, u_{2} \in \mathbb{Z}_{p}$ e $r_{1}, r_{2} \in S_{i}$, com $r_{1} r_{2}=r_{2} r_{1}$, tal que $s_{1}=\nu\left(u_{1}, r_{1}\right)$ e $s_{2}=\nu\left(u_{2}, r_{2}\right)$. Então;

$s_{1} s_{2} \neq s_{2} s_{1}$

$\nu\left(u_{1}, r_{1}\right) . \nu\left(u_{2}, r_{2}\right) \neq \nu\left(u_{2}, r_{2}\right) . \nu\left(u_{1}, r_{1}\right)$,

$\nu\left(\left(u_{1}, r_{1}\right) \cdot\left(u_{2}, r_{2}\right) \cdot\left(u_{1}, r_{1}\right)^{-1} \cdot\left(u_{2}, r_{2}\right)^{-1}\right) \neq e$

$\nu\left(u^{\prime}, r_{1} r_{2} r_{1}^{-1} r_{2}^{-1}\right) \neq e$, para algum $u^{\prime} \in \mathbb{Z}_{p}$

$\nu\left(u^{\prime}, e\right) \neq e$

Daí, $u^{\prime} \neq e$ e $\left(u^{\prime}, e\right) \in\left(\mathbb{Z}_{p} \otimes S\right)^{\prime} \cap\left(\mathbb{Z}_{p} \otimes S_{0}\right)$. Desde que a ordem de $\mathbb{Z}_{p} \otimes S_{0}$ é $p$, temos que $\mathbb{Z}_{p} \otimes S_{0} \subset\left(\mathbb{Z}_{p} \otimes S\right)^{\prime}$. (a) Se $S_{1}=S_{0}$

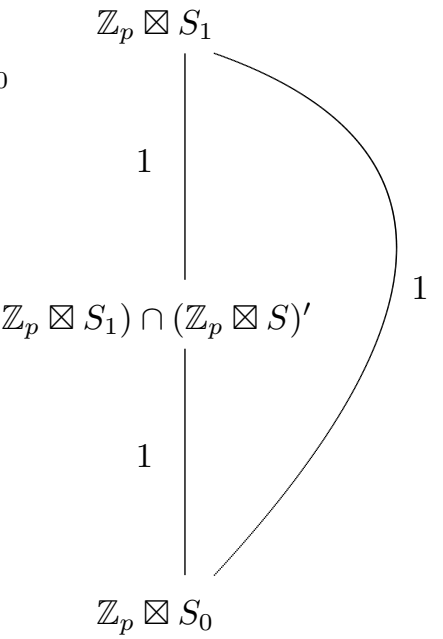

(b) Se $S_{1} \neq S_{0}$

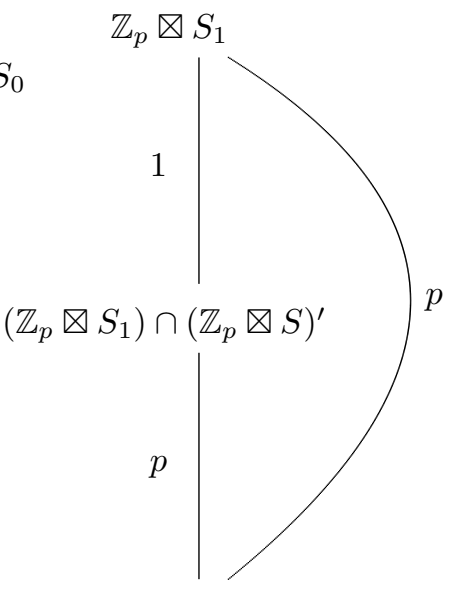

$\mathbb{Z}_{p} \otimes S_{0}$

Fig. 4. A interseção $\left(\mathbb{Z}_{p} \otimes S_{1}\right) \cap\left(\mathbb{Z}_{p} \otimes S\right)^{\prime}$ quando $\mathbb{Z}_{p} \otimes S_{0} \subset\left(\mathbb{Z}_{p} \otimes S\right)^{\prime}$

Pelo lema 3, $\left(\mathbb{Z}_{p} \otimes S_{i}\right) \subset\left(\mathbb{Z}_{p} \otimes S\right)^{\prime}$ e $S_{i} \subset S^{\prime}$, para cada $i$. Portanto $S_{i}$ é um grupo abeliano ou $S_{i} \subset S^{\prime}$.

Suponha agora que não temos informação acerca da ordem de $\mathbb{Z}_{p} \otimes S$, isto é, não possamos usar a hipótese de $\mathbb{Z}_{p} \otimes S$ ser um $p$-grupo. Neste caso temos que $S$ deve ser um grupo finito e genérico. Trabalhando, outra vez, com a família $\left\{S_{i}\right\}$ definida na equação (4) mostraremos que quando $U=\mathbb{Z}_{p}$, cada $S_{i}$ deve ser um $p$-grupo. Em esta direção começamos mostrando um resultado sobre um importante subgrupo normal do grupos dos estados $S$. Este subgrupo é o conjunto dos estados de partida das transições que chegam no estado neutro $e$. Ou de uma maneira mais formal é o conjunto de estados resultantes da segunda projeção sobre o kernel de $\nu$;

$$
S_{d}=\left\{s \in S ; \nu(u, s)=e \text { for some } u \in \mathbb{Z}_{p}\right\}
$$

Notemos que este subgrupo normal também é isomorfo com $\mathbb{Z}_{p}$ e;

Lema 5: Considere o codificador $\nu$, $\omega$, e $\mathbb{Z}_{p} \otimes S$ da Definição 2. Além disso considere o subgrupo $S_{d}$ definido na equação (6), então; 
1) Se existir $s \neq e$ e $s \in S_{d} \cap S_{i}$ então $S_{d} \subset S_{i}$, para $i \geq 0$

2) Se $S_{d} \subset S_{i}$ então $\nu\left(\mathbb{Z}_{p}, S_{d}\right) \subset S_{i}$, para $i \geq 0$.

Prova.-

1) Desde que $p \in S_{d} \cap S_{i}$, então $\left\{s, s^{2}, \ldots, s^{p-1}, s^{p}=\right.$ e\} $\subset S_{d} \cap S_{i}$.

2) Dado $r \neq e$ tal que $r \in S_{i} \cap S_{d}$ suponha que existe algum $u \in \mathbb{Z}_{p}$ tal que $\nu(u, r)=s \notin S_{i}$. Para o subgrupo $S_{1}=\left\{s_{0}, s_{1}=\nu\left(u_{1}, e\right), s_{2}=\nu\left(u_{2}, e\right), \ldots, s_{p-1}=\right.$ $\left.\nu\left(u_{p-1}, e\right)\right\}$, temos que $s S_{1}$ é um coset onde cada elemento é $\nu(u, r) \nu\left(u_{i}, e\right)=\nu\left(u^{\prime}, r\right)$, para algum $u^{\prime} \in \mathbb{Z}_{p}$. Daí $s S_{1}=\left\{\nu\left(\mathbb{Z}_{p}, r\right)\right\}$ com $s S_{1} \cap S_{i}=\emptyset$. Mas, desde que $r \in S_{d}$, existe pelo menos $u_{0} \in \mathbb{Z}_{p}$ tal que $\nu\left(u_{0}, r\right)=e$ em contradição com $s S_{1} \cap S_{i}=\emptyset$.

Teorema 2: Considere o codificador $\nu, \omega$, e $\mathbb{Z}_{p} \otimes S$ da Definição 2, onde $p$ é primo. Então cada $S_{i}$ de (4) deve ser um $p$-grupo

Por indução sobre $i$. Para $i=1$ temos $\left[S_{1}: S_{0}\right]=p$ ou $\left[S_{1}: S_{0}\right]=1$. Agora suponha que existe um número natural $k>1$ tal que $\left[S_{i}: S_{i-1}\right]=p$, para cada $i \leq k$. Temos que o subgrupo $S_{k}$ tem $p^{k}$ elementos e cada um dos seus elementos possui ordem $p^{i}, i \leq k$. Se $p>\left[S_{k+1}: S_{k}\right]>1$ então $\left[S_{k+1}: S_{k}\right]=m=q_{1}^{r_{1}} q_{2}^{r_{2}} \ldots q_{t}^{r_{t}}$, onde cada $q_{i}$ é um primo com $q_{i}<p$. Deve existir um elemento $s \in\left(S_{k+1}-S_{k}\right)$ tal que $s^{q_{1}}=e$.

Sejam $u \in \mathbb{Z}_{p}$ e $r \in S_{k}$ tais que $\nu(u, r)=s$, então $\nu\left(u_{1}, r^{q_{1}}\right)=e$. Daí $r^{q_{1}} \in S_{d} \cap S_{k}$.

Se $r \neq e$ então $r^{q_{1}} \neq e$, pois $q_{1}<p$. Pelo Lema $5, S_{d} \subset S_{k}$ e $\nu(u, r)=s \in S_{k}$, uma contradição.

Se $r=e$ então $\nu(u, r)=s \in S_{1} \subset S_{k}$, também uma contradição.

Teorema 3: Considere o codificador $\nu$, $\omega$, e $\mathbb{Z}_{p} \otimes S$ da Definição 2 , onde $\mathbb{Z}_{p} \otimes S$ é não abeliano e $p$ é um primo positivo, então

1) Se $S$ é abeliano então o código tem transições paralelas.

2) Se $S$ é não abeliano então o código é não controlável

\section{Prova.-}

1) Pelo Lema 2

2) Se $S$ não é um $p$-grupo então pelo Teorema 2 o código resultante é não-controlável. Se $S$ é um $p$-grupo, então $\mathbb{Z}_{p} \otimes S$ é também um $p$-grupo, então pelo Lema $4 S$ é abeliano, uma contradição.

\section{CONClusões}

Mostramos que codificadores convolucionais generalizados definidos sobre extensões não-abelianas $Z_{p} \otimes S, p$ primo não produzem bons códigos. Fica pendente obter resultados sobre extensões não abelianas $\mathbb{Z}_{p^{n}} \otimes S$ ou $\left(\mathbb{Z}_{p}\right)^{n} \otimes S$.

\section{REFERÊNCIAS}

[1] G.D. Forney and M.D. Trott. The dynamics of group codes; state spaces, trellis diagrams and canonical encoders. IT 39(5):1491-1513, 1993.

[2] Shu Lin and Daniel J. Costello. Error Control Coding: Fundamentals and Applications. Prentice-Hall, New Jersey, 1983.

[3] Gottfried Ungerboeck. Channel coding with multilevel-phase signals. IEEE Transactions on Information Theory, 28:55-67, 1982.

[4] H.A. Loeliger. Signal sets matched to groups. IEEE Trans. Inform. Theory, 37:1675-1682, November 1991.
[5] H. A. Loeliger and T. Mittelholzer. Convolutional codes over groups. IEEE Transactions on Information Theory, 42:1659-1687, 1996.

[6] Douglas Lind and Brian Marcus. An Introduction to Symbolic Dynamics and Coding. Cambridge University Press, New York, 1995.

[7] Jan C. Willems. Models for dynamics. In H. O. Walther U. Kirchgraber, editor, Dynamics Reported, volume 2, pages 171-269. Wiley and Teubner, 1989.

[8] Joseph J. Rotman. An Introduction to the Theory of the Groups. Springer Verlag, New York, fourth edition, 1995.

[9] Marshall Hall. The Theory of Groups. Mac Millan, New York, 1959.

[10] Fagnani F. and Zampieri S. Minimal syndrome formers for group codes. IEEE Transactions on Information Theory, 45(01):3-31, 1999.

[11] David G. Forney. On the hamming distance properties of group codes. IEEE Transactions on Information Theory, 38:1797-1801, 1992

[12] R James. The groups of order $p^{6}$ ( $p$ an odd order prime). Math. Comput., 34:613-637, 1980.

[13] E. A. O'Brien. The groups of order 256. Journal of Algebra, 143:219235, 1991.

[14] The GAP Group. GAP - Groups, Algorithms, and Programming, Version 4.4, 2005. (http://www.gap-system.org).

[15] Yff P. On $k$-conjugacy in a group. Proc. Edimburg Math. Soc., 2:14:1-4, $1964 / 65$. 